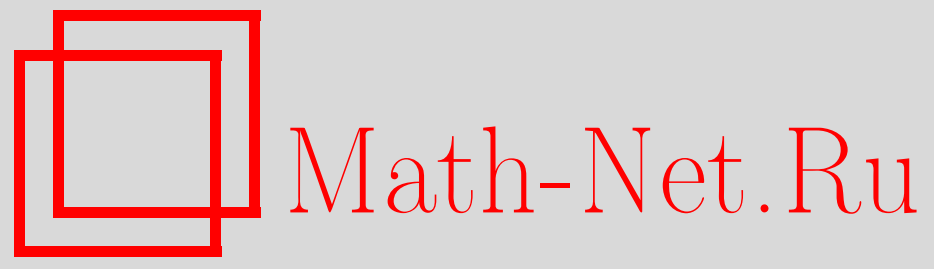

А. Н. Фролов, Универсальные предельные теоремы для приращений процессов с независимыми приращениями, Теория вероятн. и ее примен., 2004, том 49, выпуск 3, 601-609

DOI: https://doi.org/10.4213/tvp211

Использование Общероссийского математического портала Math-Net.Ru подразумевает, что вы прочитали и согласны с пользовательским соглашением

http://www . mathnet.ru/rus/agreement

Параметры загрузки:

IP : 54.84 .234 .179

26 апреля 2023 г., 13:39:37

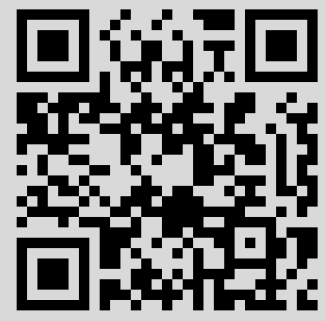


7. Фельдман Г. М. Теорема Скитовича-Дармуа для дискретных периодических абелевых групп. - Теория вероятн. и ее примен., 1997, т. 42, в. 4, с. 747-756.

8. Фельдман Г. М. К теореме Скитовича-Дармуа для конечных абелевых групп. Теория вероятн. и ее примен., 2000 , т. 45 , в. 3 , с. 603-607.

9. Feldman G. M., Graczyk P. On the Skitovich-Darmois theorem on compact Abelian groups. - J. Theoret. Probab., 2000, v. 13, № 3, p. 859-869.

Поступила в редакцию

11.VI.2002

(c) $2004 \mathrm{r}$.

ФРОЛОВ А. Н.*

\title{
УНИВЕРСАЛЬНЫЕ ПРЕДЕЛЬНЫЕ ТЕОРЕМЫ ДЛЯ ПРИРАЩЕНИЙ ПРОЦЕССОВ С НЕЗАВИСИМЫМИ ПРИРАЩЕНИЯМИ ${ }^{1)}$
}

\begin{abstract}
Построена единая теория, описывающая п.н. (почти наверное) поведение приращений стохастически непрерывных однородных процессов с независимыми приращениями. Эта теория включает в себя усиленный закон больших чис ел, закон Эрдёша-Реньи, закон Шеппа, закон ЧёргёРевеса и закон повторного логарифма. Область применимости результатов о поведении прирашений расширена с нескольких частных случаев до всего класса стохастически непрерывных однородных процессов с независимыми приращениями.
\end{abstract}

Ключевые слова и фразы: приращения процессов с независимыми прирашениями, законы Эрдёша-Реньи и Шеппа, закон больших чисел, закон повторного логарифма.

1. Введение и основные результаты. Пусть $\xi(t), t \geqslant 0$, - стохастически непрерывный однородный процесс с независимыми приращениями, $\mu=\mathbf{E} \xi(1)$, $0 \leqslant \mu<\infty$. Пусть $\xi(t)$ непрерывен справа и имеет пределы слева. Пусть $a_{T}-$ неубываюшая непрерывная функция такая, что $T / a_{T}$ не убывает и $0<a_{T} \leqslant T$. Обозначим

$$
U_{T}=\sup _{0 \leqslant t \leqslant T-a_{T}}\left(\xi\left(t+a_{T}\right)-\xi(t)\right) .
$$

(Отметим, что $U_{T}=\xi(T)$ при $a_{T}=T$.)

В настоящей работе мы опишем класс нормирующих (неслучайных) функций $b_{T}$, для которых либо $\lim \sup \left(U_{T} / b_{T}\right)=1$ п.н., либо последнее соотношение выполнено с заменой limsup на lim, если это возможно. (Всюду, где использованы символы $\limsup , \lim \inf , \lim , O, o, \rightarrow$, считаем, что $T \rightarrow \infty$, если не оговорено противное.) Мы будем предполагать, что $b_{T}$ положительна и не убывает. В дальнейшем мы не будем различать между собой нормировки, эквивалентные на бесконечности.

Поведение однородных процессов с независимыми прирашениями во многом сходно с поведением сумм независимых одинаково распределенных (н.о.р.) случайных величин. Поэтому мы сначала опишем результаты для приращений сумм. Первые подобные результаты были получены в работах Шеппа [1] и Эрдёша и Реньи [2], изучавших асимптотическое поведение $S_{n+a_{n}}-S_{n}$ и $\max _{0 \leqslant k \leqslant n-a_{n}}\left(S_{k+a_{n}}-S_{k}\right)$ соответственно. Здесь $S_{k}$ обозначает сумму первых $k$ величин из последовательности н.о.р. случайных величин. В работе [2] и в статьях Ш. Чёргё [3], Девельса и Девроя [4] и Мейсона [5] было изучено поведение малых $\left(a_{n}=O(\ln n)\right.$ при $\left.n \rightarrow \infty\right)$ приращений

* С.-Петербургский государственный университет, математико-механический факультет, Университетский пр., 28, Старый Петергоф, 198504 С.-Петербург, Россия; e-mail: Andrei.Frolov@pobox.spbu.ru

1) Работа выполнена при поддержке грантов Е02-1.0-56 Минобразования РФ, 02-01-00779 РФФН и HUI-2258.2003.1. 
сумм. Случай больших $\left(a_{n} / \ln n \rightarrow \infty\right.$ при $\left.n \rightarrow \infty\right)$ прирашений был исследован М. Чёргё и Ревесом [6] для случайных величин с конечным вторым моментом. Оказалось, что в предельных теоремах о п.н. поведении малых прирашений нормирующая последовательность существенно зависит от распределения слагаемых, а иногда даже однозначно определяет это распределение. Этот эффект был открыт Эрдёшем и Реньи [2]. Результаты для малых приращений называются законами Эрдёша-Реньи. Если речь идет о статистике $S_{n+a_{n}}-S_{n}$, говорят о законе Шеппа. В случае больших приращений нормируюшая последовательность универсальна и совпадает с нормировкой для нормального распределения с теми же средним и дисперсией, что и у слагаемых. Далее эти результаты мы называем законами Чёргё-Ревеса. Отметим, что случаю $a_{n}=n$ соответствует известный закон повторного логарифма (ЗПЛ).

В работах автора [7]-[9] найдена формула универсальной нормирующей последовательности в предельных теоремах для приращений сумм. По этой формуле вычисляется нормируюшая последовательность в усиленном законе больших чисел (УЗБЧ), законе Эрдёша-Реньи, законе Шеппа, законе Чёргё-Ревеса и ЗПЛ.

Асимптотическое поведение приращений однородных процессов с независимыми приращениями было исследовано лишь для некоторых важных частных случаев. М. Чёргё и Ревес [6] исследовали поведение приращений винеровского процесса. Результаты о поведении прирашений пуассоновского процесса являются частным случаем соответствующих результатов для процессов восстановления и могут быть найдены в работах Девельса и Штайнебаха [10], Штайнебаха [11] и в статьях из библиографий этих работ. Асимптотика прирашений устойчивых процессов со скачками одного знака была исследована Н. М. Зинченко [12].

В настоящей работе мы получим формулу универсальной нормирующей функции в предельных теоремах о п.н. поведении приращений однородных процессов с независимыми приращениями. Это позволяет охватить единой формулировкой УЗБЧ, закон Эрдёша-Реньи, закон Шеппа, закон Чёргё-Ревеса и ЗПЛ для этих процессов. Результаты для приращений винеровского и пуассоновского процессов являются следствиями наших теорем, а наши результаты для устойчивых процессов со скачками одного знака дополняют и усиливают результаты работы [12]. Кроме того, существенно расширяется класс процессов, к которым применимы соответствующие теоремы. Среди новых примеров - обобщенные пуассоновские процессы. Напомним, что обобщенный пуассоновский процесс - это сумма случайного числа н.о.р. случайных величин, где число слагаемых определяется пуассоновским процессом, независимым от слагаемых. Такие процессы возникают, например, в различных моделях страховой и финансовой математики, где они описывают процесс выплат по страховому портфелю. В наших примерах на слагаемые могут накладываться различные ограничения. В частности, распределения слагаемых могут принадлежать областям притяжения нормального закона и асимметричных устойчивых законов с показателем $\alpha \in(1,2)$. Из приведенных примеров можно построить много новых, рассматривая, например, линейные комбинации независимых процессов, указанных выше.

Наряду с $U_{T}$ мы будем рассматривать

$$
\begin{aligned}
W_{T} & =\sup _{0 \leqslant t \leqslant T-a_{T}} \sup _{0 \leqslant s \leqslant a_{T}}(\xi(t+s)-\xi(t)), \\
Q_{T} & =\xi\left(T+a_{T}\right)-\xi(T), \quad R_{T}=\xi(T)-\xi\left(T-a_{T}\right) .
\end{aligned}
$$

Отметим, что $W_{T}=\sup _{0 \leqslant s \leqslant T} \xi(s), R_{T}=\xi(T)$ при $a_{T}=T$.

Обозначим $h_{0}=\sup \left\{h: \varphi_{\xi(1)}(h)=\mathbf{E} e^{h \xi(1)}<\infty\right\}$.

Если $h_{0}>0$, то положим

$$
\begin{aligned}
\zeta_{\xi(1)}(z) & =\sup \left\{z h-\ln \varphi_{\xi(1)}(h): h \geqslant 0, \varphi_{\xi(1)}(h)<\infty\right\}, \\
\gamma_{\xi(1)}(x) & =\sup \left\{z: \zeta_{\xi(1)}(z) \leqslant x\right\}, \\
\lambda_{T}(x) & =\gamma_{\xi(1)}(x) \quad \text { для всех } \quad T \geqslant 0 .
\end{aligned}
$$

Для краткости записи в этом случае обозначим $\zeta(z)=\zeta_{\xi(1)}(z), \gamma(x)=\gamma_{\xi(1)}(x)$.

Функция $\zeta(z)$ хорошо известна из теории вероятностей больших уклонений (см., например, книгу А. А. Боровкова [13]). Она называется функцией уклонений и равна логарифму функции Чернова, взятому с обратным знаком. 
Если $h_{0}=0$, то мы определим функции $\lambda_{T}(x)$ для последовательности усечений $\xi(1)$ на возрастающих уровнях. Для этого возьмем непрерывную функцию $y_{T}$ такую, что $y_{T} \nearrow \infty$, и для всех $T \geqslant 0$ обозначим

$$
\xi_{1}(T)=\min \left\{\xi(1), y_{T}\right\}, \quad \eta(T)= \begin{cases}\xi_{1}(T)-\mathbf{E} \xi_{1}(T), & \text { если } \quad \mu=0 \\ \xi_{1}(T), & \text { если } \quad \mu>0 .\end{cases}
$$

Пусть $\lambda_{T}(x)=\gamma_{\eta(T)}(x)$ для всех $T \geqslant 0$, где функция $\gamma_{\eta(T)}(x)$ определена по формулам (1), (2) с заменой $\xi(1)$ на $\eta(T)$.

Положим

$$
b_{T}=a_{T} \lambda_{T}\left(\frac{\beta_{T}}{a_{T}}\right), \quad \text { где } \quad \beta_{T}=\ln \frac{T}{a_{T}}+\ln \ln T .
$$

Эта последняя формула, фактически, и есть основной результат этой работы. Ниже мы убедимся, что во всех упомянутых выше предельных теоремах нормируюшая функция вычисляется по этой формуле.

Сформулируем теперь наши результаты.

Теорема 1. Предположим, ито $b_{T}$ эквивалентна неубывающей функиии и

$$
\underset{\theta \searrow 1}{\limsup } \limsup _{T \rightarrow \infty} \frac{b_{\theta T}}{b_{T}}=1 .
$$

Пусть выполнено одно из следуюших условий:

1) $h_{0}>0$

2) $a_{T} \rightarrow \infty, \sum_{n} \mathbf{P}\left\{\xi(1) \geqslant b_{n}\right\}<\infty$ и неравенство

$$
\mathbf{P}\left\{\xi\left(\left[(1+\varepsilon) a_{T}\right]\right) \geqslant(1+\varepsilon) b_{T}\right\} \leqslant H_{1} e^{-(1+\tau) \beta_{T}}+H_{2} a_{T} \mathbf{P}\left\{\xi(1) \geqslant b_{T}\right\}
$$

имеет место для всех положительных $\varepsilon$ u $\tau$, некоторых $H_{1}=H_{1}(\varepsilon, \tau)>0 u$ $H_{2}=H_{2}(\varepsilon, \tau) \geqslant 0$ и всех достаточно больших $T$ (здесь и далее [.] обозначает челую часть числа в скобках).

Пусть для любого $\varepsilon>0$ существует $q \in(0,1)$ такое, ито для всех $t \leqslant(1+\varepsilon) a_{T}$ неравенство

$$
\mathbf{P}\left\{\xi(t) \geqslant-\varepsilon b_{T}\right\} \geqslant q
$$

выполнено при всех достаточно больших $T$.

Тогда

$$
\limsup \frac{W_{T}}{b_{T}} \leqslant 1 \quad \text { п.н. }
$$

Теорема 2. Пусть неравенство

$$
\mathbf{P}\left\{\xi\left(a_{T}\right) \geqslant(1-\varepsilon) b_{T}\right\} \geqslant H_{3} e^{-(1-\tau) \beta_{T}}
$$

выполнено для всех достаточно малых положительных $\varepsilon$ и $\tau$ и всех достаточно больших T. $u(5)$.

Если $a_{T} / T \rightarrow 1$, то предположим дополнительно, что выполнены условия (3)

Tогда

$$
\limsup \frac{R_{T}}{b_{T}} \geqslant 1 \quad \text { n.н. }
$$

$B$ последнем соотношении $R_{T}$ можно заменить на $Q_{T}$.

Проверку условий (5) и (7) облегчает следующее предложение.

Предложение 1. 1) Если $a_{T}>1$ u $b_{T} \rightarrow \infty$, то неравенство (5) можно проверять только для $t=\left[(1+\varepsilon) a_{T}\right]$.

2) Eсли $b_{T} \rightarrow \infty$, то в неравенстве (7) можно заменить $\xi\left(a_{T}\right)$ на $\xi\left(\left[a_{T}\right]\right)$.

Теорема 3. Предположим, что $b_{T}$ эквивалентна неубываюшей функиии, $b_{T} \rightarrow \infty$ и выполнено условие (3). Пусть неравенство (7) выполнено для всех достаточно мальх положительных $\varepsilon$ u $\tau$ и всех достаточно больших $T$. Пусть $\ln \ln T=o\left(\ln \left(T / a_{T}\right)\right)$.

тогда

$$
\liminf \frac{U_{T}}{b_{T}} \geqslant 1 \quad \text { п.н. }
$$


Из теорем $1-3$ и неравенств $R_{T} \leqslant U_{T} \leqslant W_{T}$ вытекает следующая теорема.

Теорема 4. Если выполнены условия теорем 1 и 2, то

$$
\limsup \frac{W_{T}}{b_{T}}=\limsup \frac{U_{T}}{b_{T}}=\limsup \frac{R_{T}}{b_{T}}=\limsup \frac{Q_{T}}{b_{T}}=1 \quad \text { п.н. }
$$

Если выполнены условия теорем 1 и 3 , то

$$
\lim \frac{W_{T}}{b_{T}}=\lim \frac{U_{T}}{b_{T}}=1 \quad \text { n.н. }
$$

Теперь мы сформулируем ряд важных следствий из теоремы 4.

Мы начнем с тех важных частных случаев, где нормируюшая функция может быть явно выписана для всего возможного диапазона изменения $a_{T}$. Это можно сделать для винеровского процесса и для устойчивых процессов без положительных скачков. В этом случае характеристическая функция $\xi(1)$ имеет вид

$$
\psi(t)=\exp \left\{i \mu t-c|t|^{\alpha}\left(1+i \frac{t}{|t|} \operatorname{tg} \frac{\pi}{2} \alpha\right)\right\}
$$

где $c=\cos (\pi(2-\alpha) / 2) / \alpha, 1<\alpha \leqslant 2$. Постоянная $c$ здесь выбрана так, чтобы при $\alpha=2$ и $\mu=0$ получалось стандартное нормальное распределение. Случай $\alpha=2$ соответствует нормальному закону с параметрами $\mu$ и 1 . Можно показать, что в рассматриваемом случае $h_{0}=+\infty$ и $\varphi_{\xi(1)}(h)=\exp \left\{\mu h+h^{\alpha} / \alpha\right\}$ при всех $h \geqslant 0$, что в силу (1), (2) дает $\gamma(x)=\mu+(\alpha x /(\alpha-1))^{(\alpha-1) / \alpha}, x \geqslant 0$. С учетом этого, мы можем сформулировать следующий результат.

Теорема 5. Пусть $\xi(1)$ имеет характеристическую функиию (11).

Тогда соотношения (9) выполнены с

$$
b_{T}=\mu a_{T}+\left(\frac{\alpha}{\alpha-1}\right)^{(\alpha-1) / \alpha} a_{T}^{1 / \alpha}\left(\ln \frac{T}{a_{T}}+\ln \ln T\right)^{(\alpha-1) / \alpha} .
$$

Если дополнительно или $\ln \ln T=o\left(\ln \left(T / a_{T}\right)\right)$, или $a_{T}=T$ и $\mu>0$, то выполнено (10).

Если $\alpha=2$ и $\mu=0$, то $\xi(t)$ - стандартный винеровский процесс и

$$
b_{T}=\sqrt{2 a_{T}\left(\ln \frac{T}{a_{T}}+\ln \ln T\right)} .
$$

Теорема 5 получена в этом случае М. Чёргё и Ревесом [6]. В частности, при $a_{T}=T$ мы получаем ЗПЛ с $b_{T}=\sqrt{2 T \ln \ln T}$. Если $\alpha<2$ и $\mu=0$, то $\xi(t)-$ устойчивый случайный процесс без положительных скачков. Для таких процессов соотношения (9) (для $U_{T}$ и $R_{T}$ ) получены Н.M. Зинченко [12], а в остальной части (за исключением УЗБЧ) результаты новые.

УЗБЧ для винеровского процесса и для устойчивых процессов без положительных скачков $(\alpha \in(1,2))$ получается из теоремы 5 при $a_{T}=T$ сначала для процессов с $\mu>0$, а затем, как следствие, для процессов с произвольным $\mu$. Общий случай мы рассмотрим ниже в теореме 12 .

Часто простая формула для нормирующей функции не может быть выписана для всех $a_{T}$. Поэтому далее мы рассмотрим отдельно поведение малых и больших приращений.

Пусть сначала $a_{T}=c \ln T, c>0$. Определим постоянную $c_{0} \geqslant 0$ соотношением $1 / c_{0}=\sup \{\zeta(z): \zeta(z)<\infty\}$. Отметим, что для некоторых распределений $c_{0}>0$ (см. [4]).

Теорема 6 (УЗБЧ Эрдёша-Реньи). Если $h_{0}>0 u a_{T}=c \ln T, c>c_{0}, m o$

$$
\lim \frac{W_{T}}{a_{T}}=\lim \frac{U_{T}}{a_{T}}=\limsup \frac{R_{T}}{a_{T}}=\limsup \frac{Q_{T}}{a_{T}}=\gamma(1 / c) \quad \text { n.н. }
$$

Пусть теперь $a_{T}=o(\ln T)$.

Теорема 7. Пусть $h_{0}>0, a_{T}=o(\ln T)$. Если выполнены условия теорем 1 и 2, то соотношения (9) выполнены с $b_{T}=a_{T} \gamma\left(\ln T / a_{T}\right)$. Если выполнены условия теорем 1 и 3, то выполнено (10). 
Здесь мы сформулировали законы Эрдёша-Реньи и Шеппа для процессов с независимыми прирашениями только для $c>c_{0}$. В работах [1]-[4] для сумм н.о.p. случайных величин были получены аналогичные результаты для всех $c>0$, а в работе Мейсона [5] был изучен случай $a_{T}=o(\ln T)$. (Для пуассоновского процесса соответствуюшие результаты можно найти в [11]. Для винеровского процесса и устойчивых процессов - см. теорему 5.) Однако условия в случаях $c \leqslant c_{0}$ и $a_{T}=o(\ln T)$ зависят от типов распределений слагаемых. Поэтому подробное описание этих случаев будет сделано в другой работе. За исключением результатов, вытекающих из теоремы 5 , результаты теорем 6 и 7 новые.

Пусть теперь $a_{T} / \ln T \rightarrow \infty$. Поведение больших приращений зависит от моментных условий на правый хвост распределения $\xi(1)$. Для того чтобы эти условия сформулировать, нам понадобятся два класса функций $\mathscr{E}$ и $\mathscr{M}_{p}$ из работы автора [9]. Обозначим

$$
\mathscr{E}=\left\{H(x): H(x) \nearrow, \frac{H(x)}{x} \searrow, \liminf _{x \rightarrow \infty} \frac{H(c x)}{H(x)}>1 \text { для некоторого } c>1\right\} .
$$

Отметим, что если $H(x) \in \mathscr{E}$, то $\ln x=o(H(x))$ при $x \rightarrow \infty$ и $\left(H^{-1}(x)\right)^{2} / x$ не убывает. (Здесь и далее $H^{-1}(x)$ обозначает обратную функцию.) Пусть

$$
\begin{aligned}
\mathscr{M}_{p}=\left\{H(x): H(x) \nearrow, \limsup _{x \rightarrow \infty} \frac{H(c x)}{H(x)}<\infty \text { для всех } c>0,\right. \\
\left.\frac{H(x)}{x^{p}} \text { не убывает и } \lim _{x \rightarrow \infty} \frac{\ln H(x)}{\ln x}=p \text { для нексторого } p>0\right\} .
\end{aligned}
$$

Например, $H(x)=x^{p}$ принадлежит классу $\mathscr{E}$ при $p \in(0,1)$ и классу $\mathscr{M}_{p}$ при $p>0$. Другие примеры можно получить умножением $x^{p}$ на медленно меняющуюся функцию.

Ясно, что результаты о поведении больших прирашений для центрированных $(\mu=0)$ процессов и для нецентрированных процессов должны сильно отличаться. Так, например, при $a_{T}=T$ для центрированных процессов мы должны получить результат типа ЗПЛ, а для нецентрированных процессов - УЗБЧ. Далее мы рассматриваем эти случаи отдельно.

Рассмотрим сначала центрированные процессы. Обозначим $\xi^{+}(1)=\max \{\xi(1), 0\}$.

Теорема 8. Пусть $\mu=0, \mathbf{E} \xi^{2}(1)=1$.

Предположим, что выполнено одно из следующих трех условий:

1) $h_{0}>0$ u $a_{T} / \ln T \rightarrow \infty$;

2) $\mathbf{E} \exp \left\{H\left(\xi^{+}(1)\right)\right\}<\infty, H(x) \in \mathscr{E}, a_{T} \geqslant C\left(H^{-1}(\ln T)\right)^{2} / \ln T$, где $C=C(H)$ абсолютная положительная постоянная;

3) $\mathbf{E} H\left(\xi^{+}(1)\right)<\infty, H(x) \in \mathscr{M}_{p}, p>2, a_{T} \geqslant C\left(H^{-1}(T)\right)^{2} / \ln T$, где $C-$ произвольная положительная постоянная.

Тогда (9) выполнено с $b_{T}$ из (13).

Если, кроме того, $\ln \ln T=o\left(\ln \left(T / a_{T}\right)\right)$, то выполнено (10).

Перейдем к случаю $\mathbf{E} \xi^{2}(1)=\infty$. Положим $F(x)=\mathbf{P}\{\xi(1)<x\}$.

В дальнейшем мы будем использовать следующие обозначения: $F \in D N(\alpha)$ $(F \in D(\alpha)), \alpha \in(1,2]$, если $F$ принадлежит области нормального (соответственно ненормального) притяжения устойчивого закона с характеристической функцией (11). В случае нормального притяжения мы считаем, что нормирующие постоянные имеют вид $n^{1 / \alpha}$. В противном случае нужно соответствующим образом отнормировать $\xi(1)$.

Ясно, что в условиях теоремы $8 F \in D N(2)$. Опишем здесь важный класс процессов, для которых $F \in D(\alpha)$.

Пусть $\left\{X_{k}\right\}$ - последовательность н.о.р. случайных величин, $\nu(t)$ - независимый от $X$ 'ов пуассоновский процесс. Положим $\xi(t)=\sum_{k=1}^{\nu(t)} X_{k}, \xi(0)=0$. Покажем, что $F \in D(\alpha)$, если $\mathbf{P}\left\{X_{1}<x\right\} \in D(\alpha)$. Действительно, характеристическая функция $\xi(1)$ равна $\exp \{(h(t)-1) \mathbf{E} \nu(1)\}$, где $h(t)-$ характеристическая функция $X_{1}$. Остается воспользоваться теоремой 2.6 .5 книги И. А. Ибрагимова и Ю. В. Линника [14, с. 103].

Далее, если $g(x)$ - правильно меняюшаяся функция, то $g^{-1}(x)$ - асимптотически обратная к ней функция, т.е. $g^{-1}(g(x)) \sim g\left(g^{-1}(x)\right) \sim x$ при $x \rightarrow 0$. 
Теорема 9. Пусть $\mu=0, F \in D(2)$. Пусть выполнено одно из условий 1)-3) теоремы 8.

Тогда (9) выполнено с $b_{T}=a_{T} m\left(f^{-1}\left(\beta_{T} / a_{T}\right)\right)$, где $m(h)=h G(1 / h), f(h)=$ $h^{2} G(1 / h) / 2, G(x)=\int_{-x}^{0} u^{2} d F(u), x>0(G(x)$ - медленно меняюшаяся функиия).

Если, кроме того, $\ln \ln T=o\left(\ln \left(T / a_{T}\right)\right)$, то выполнено (10).

Перейдем к случаю $\alpha \in(1,2)$.

Теорема 10. Пусть $\mu=0 u F \in D N(\alpha), \alpha \in(1,2)$.

Предположим, что выполнено одно из следующих трех условий:

1) $h_{0}>0 u a_{T} / \ln T \rightarrow \infty$;

2) $\mathrm{E} \exp \left\{H\left(\xi^{+}(1)\right)\right\}<\infty, H(x) \in \mathscr{E}, a_{T} \geqslant C\left(H^{-1}(\ln T)\right)^{\alpha} /(\ln T)^{\alpha-1}$, где $C=$ $C(H)$ - абсолютная положительная постоянная;

3) $\mathrm{E} H\left(\xi^{+}(1)\right)<\infty, H(x) \in \mathscr{M}_{p}, p>\alpha, a_{T} \geqslant C\left(H^{-1}(T)\right)^{\alpha} /(\ln T)^{\alpha-1}$, aде $C$ произвольная положительная постоянная.

Тогда (9) выполнено $с b_{T}$ из (12).

Если, кроме того, $\ln \ln T=o\left(\ln \left(T / a_{T}\right)\right)$, то выполнено (10).

Теорема 11. Пусть $\mu=0, F \in D(\alpha), \alpha \in(1,2)$. Положим $b_{T}=$ $a_{T} m\left(f^{-1}\left(\beta_{T} / a_{T}\right)\right)$, где $m(h)=h^{\alpha-1} G(1 / h) \alpha \Gamma(2-\alpha) /(\alpha-1), f(h)=\Gamma(2-\alpha) h^{\alpha} G(1 / h)$, $G(x)=x^{\alpha} F(-x), x>0 .(G(x)-$ медленно меняющаяся функиия.)

Предположим, что выполнено одно из следуюиих трех условий:

1) $h_{0}>0 u a_{T} / \ln T \rightarrow \infty$;

2) $\mathbf{E} \exp \left\{H\left(\xi^{+}(1)\right)\right\}<\infty, H(x) \in \mathscr{E}, b_{T} \geqslant C H^{-1}(\ln T)$, где $C=C(H)-$ абсолютная положительная постоянная;

3) $\mathbf{E} H\left(\xi^{+}(1)\right)<\infty, H(x) \in \mathscr{M}_{p}, p>\alpha, b_{T} \geqslant C H^{-1}(T)$, где $C-$ произвольная положительная постоянная.

Тогда выполнено (9).

Если, кроме того, $\ln \ln T=o\left(\ln \left(T / a_{T}\right)\right)$, то выполнено (10).

Результаты теорем 8-11 новые. Для н.о.р. случайных величин результаты, аналогичные теоремам 8-11, получены автором [9].

При $a_{T}=T$ из теорем 8-11 вытекает ЗПЛ для процессов с независимыми прирашениями.

В заключение рассмотрим случай $\mu>0$.

Теорема 12. Пусть выполнень условия одной из теорем 8-11 и $\mu>0$. Тогда (9) выполнено с $b_{T}=\mu a_{T}$.

Если, кроме того, $\ln \ln T=o\left(\ln \left(T / a_{T}\right)\right)$ или $a_{T}=T$, то выполнено (10).

Из теоремы 12 при $a_{T}=T$ следует УЗБЧ для процессов с $\mu>0$. Применяя этот результат к процессу $\xi(t)-\mu t+t$, получим УЗБЧ и в случае $\mu \leqslant 0$.

2. Доказательства. Мы будем использовать следующую лемму.

Лемма 1. Пусть $\xi(t), t \geqslant 0,-$ однородный прочесс с независимьми приращениями, $r, c \geqslant 0, q>0$. Если $\mathbf{P}\{\xi(t) \geqslant-c\} \geqslant q$ для всех $t \leqslant T$, mo

$$
\mathbf{P}\left\{\sup _{0 \leqslant s \leqslant t \leqslant T}(\xi(t)-\xi(s)) \geqslant r\right\} \leqslant q^{-2} \mathbf{P}\{\xi(T) \geqslant r-c\} .
$$

Лемма 1 следует из аналогичного результата для сумм н.о.р. случайных величин и сепарабельности процесса $\xi(t)$.

Д о к а з а т е л ь с т в о т е о р е м ы 1. Предположим, что выполнено условие 2). Возьмем $\varepsilon>0$. Положим $u_{T}=\varepsilon a_{T}-\left\{(1+\varepsilon) a_{T}\right\}$, где $\{\cdot\}$ обозначает дробную часть числа в скобках. По лемме 1 имеем

$$
\begin{aligned}
P_{T} & =\mathbf{P}\left\{W_{T} \geqslant(1+2 \varepsilon) b_{T}\right\} \\
& \leqslant \mathbf{P}\left\{\bigcup_{j=1}^{\left[T / u_{T}\right]}\left\{\sup _{(j-1) u_{T} \leqslant t \leqslant j u_{T}} \sup _{0 \leqslant s \leqslant a_{T}}(\xi(t+s)-\xi(t)) \geqslant(1+2 \varepsilon) b_{T}\right\}\right\} \\
& \leqslant \frac{T}{u_{T}} \mathbf{P}\left\{\sup _{0 \leqslant t \leqslant u_{T}} \sup _{0 \leqslant s \leqslant a_{T}}(\xi(t+s)-\xi(t)) \geqslant(1+2 \varepsilon) b_{T}\right\} \\
& \leqslant \frac{T}{q^{2} u_{T}} \mathbf{P}\left\{\xi\left(\left[(1+\varepsilon) a_{T}\right]\right) \geqslant(1+\varepsilon) b_{T}\right\}
\end{aligned}
$$


для всех достаточно больших T. В силу условия 2)

$$
\begin{aligned}
P_{T} & \leqslant \frac{T}{q^{2} u_{T}}\left(H_{1} \exp \left\{-(1+\tau) \beta_{T}\right\}+H_{2} a_{T} \mathbf{P}\left\{\xi(1) \geqslant b_{T}\right\}\right) \\
& \leqslant C_{1}(\ln T)^{-(1+\tau)}+C_{2} T \mathbf{P}\left\{\xi(1) \geqslant b_{T}\right\}
\end{aligned}
$$

для всех достаточно больших $T$.

Пусть $\theta>1$. Положим

$$
n_{k}=\min \left\{n: \theta^{k-1}<n \leqslant \theta^{k}, n \mathbf{P}\left\{\xi(1) \geqslant b_{n}\right\}=\min _{\theta^{k-1}<m \leqslant \theta^{k}} m \mathbf{P}\left\{\xi(1) \geqslant b_{m}\right\}\right\},
$$

$k=1,2, \ldots$ Тогда ряд $\sum_{k} P_{n_{k}}$ сходится (см. [9, с. 350]). По лемме Бореля-Кантелли $\lim \sup _{k \rightarrow \infty} W_{n_{k}} / b_{n_{k}} \leqslant 1+2 \varepsilon$ п.н. Отсюда, из (3) и того факта, что $W_{T}$ не убывает, следует (6).

Пусть теперь $h_{0}>0$. Положим $\varphi_{\xi(t)}(h)=\mathbf{E} \exp \{h \xi(t)\}$. Используя представление характеристической функции $\xi(t)$ (см., например, книгу И. И. Гихмана и А.В. Скорохода $[15$, с. 417$])$ и ее аналитичность в полосе $0>\operatorname{Im} z>-h_{0}$, нетрудно показать, что $\varphi_{\xi(t)}(h)=\left(\varphi_{\xi(1)}(h)\right)^{t}$. Определим функции $\zeta_{\xi(t)}(z)$ и $\gamma_{\xi(t)}(x)$ по формулам $(1),(2)$ с заменой $\xi(1)$ на $\xi(t)$. Тогда $\zeta_{\xi(t)}(z)=t \zeta(z / t)$ и $\gamma_{\xi(t)}(x)=t \gamma(x / t)$.

Возьмем $\varepsilon>0$. Положим $u_{T}=\varepsilon a_{T}$. Действуя так же, как при доказательстве (14), получим

$$
P_{T} \leqslant \frac{T}{q^{2} u_{T}} \mathbf{P}\left\{\xi\left((1+\varepsilon) a_{T}\right) \geqslant(1+\varepsilon) b_{T}\right\}
$$

для всех достаточно больших $T$. По неравенству Чебышева

$$
\begin{aligned}
P_{T} & \leqslant \frac{T}{q^{2} u_{T}} \exp \left\{-\zeta_{\xi\left((1+\varepsilon) a_{T}\right)}\left((1+\varepsilon) b_{T}\right)\right\} \\
& =\frac{T}{q^{2} u_{T}} \exp \left\{-(1+\varepsilon) a_{T} \zeta\left(\frac{b_{T}}{a_{T}}\right)\right\}=\frac{T}{q^{2} u_{T}} \exp \left\{-(1+\varepsilon) \beta_{T}\right\}
\end{aligned}
$$

для всех достаточно больших $T$. Далее доказательство не отличается от доказательства в случае 2).

Дока за т ель с т в о т е о р е мы 2. Возьмем $\varepsilon \in(0,1)$. В силу $(7)$

$$
P_{T}=\mathbf{P}\left\{R_{T} \geqslant(1-\varepsilon) b_{T}\right\}=\mathbf{P}\left\{\xi\left(a_{T}\right) \geqslant(1-\varepsilon) b_{T}\right\} \geqslant H_{3} \exp \left\{-(1-\tau) \beta_{T}\right\}
$$

для всех достаточно больших $T$.

Пусть $a_{T} / T \rightarrow \rho \in[0,1)$. Положим $n_{1}=1, n_{k+1}=\min \left\{n: n>n_{k}, n-a_{n} \geqslant n_{k}\right\}$. Так же, как в $\left[7\right.$, с. 164], докажем, что ряд $\sum_{k} P_{n_{k}}$ расходится. Так как события $\left\{R_{n_{k}} \geqslant(1-\varepsilon) b_{n_{k}}\right\}$ независимы, то по лемме Бореля-Кантелли мы получаем (8).

Пусть теперь $a_{T} / T \rightarrow 1$. Возьмем $\theta>1$ и положим $n_{k}=\left[\theta^{k}\right], A_{k}=\left\{\xi\left(n_{k}\right)-\right.$ $\left.\xi\left(n_{k-1}\right) \geqslant(1-2 \varepsilon) b_{n_{k}}\right\}, D_{k}=\left\{\xi\left(n_{k-1}\right)-\xi\left(n_{k}-a_{n_{k}}\right) \geqslant-\varepsilon b_{n_{k}}\right\}, k=1,2, \ldots$. Отметим, что $a_{n_{k}} \sim\left(n_{k}-n_{k-1}\right) \theta /(\theta-1)$ при $k \rightarrow \infty$. Отсюда и из (5) следует, что $\mathbf{P}\left(D_{k}\right) \geqslant q$ при всех достаточно больших $k$.

Следующие пары событий независимы: $A_{k}$ и $D_{k}, A_{k}$ и $D_{k} \overline{A_{k-1} D_{k-1}}, A_{k}$ и $D_{k} \frac{\text { A } A_{k-1} D_{k-1} A_{k-2} D_{k-2}}{2} \ldots$.

Выберем $T_{k}$ так, чтобы $a_{T_{k}}=n_{k}-n_{k-1}, k=1,2, \ldots$ Тогда $T_{k} \sim a_{T_{k}} \sim n_{k}(\theta-1) / \theta$ при $k \rightarrow \infty$. Если $\theta$ было выбрано достаточно большим, то $(\theta-1) / \theta$ близко к 1 и в силу (3) для любого $\rho>0$ неравенство $b_{n_{k}} \leqslant(1+\rho) b_{T_{k}}$ выполнено для всех достаточно больших $k$. Возьмем $\rho$ таким, чтобы $(1-2 \varepsilon)(1+\rho)=1-\varepsilon$. Тогда, принимая во внимание (7), получаем

$$
\begin{aligned}
\mathbf{P}\left(A_{k}\right) & \geqslant \mathbf{P}\left\{\xi\left(n_{k}-n_{k-1}\right) \geqslant(1-2 \varepsilon) b_{n_{k}}\right\} \geqslant \mathbf{P}\left\{\xi\left(a_{T_{k}}\right) \geqslant(1-\varepsilon) b_{T_{k}}\right\} \\
& \geqslant H_{3} \exp \left\{-(1-\tau) \beta_{T_{k}}\right\}
\end{aligned}
$$

для всех достаточно больших $k$. Отсюда следует, что ряд $\sum_{k} \mathbf{P}\left(A_{k}\right)$ расходится.

По лемме 5 на с. 267 книги В.В. Петрова [16], $\mathbf{P}\left(A_{k} D_{k}\right.$ б.ч. $) \geqslant q>0$. Следовательно, $\mathbf{P}\left\{R_{n_{k}} \geqslant(1-3 \varepsilon) b_{n_{k}}\right.$ б.ч. $\}>0$. В силу закона «нуля или единицы» Колмогорова последняя вероятность равна 1 , и теорема 2 доказана для $R_{T}$. Для $Q_{T}$ теорема доказывается аналогично. 
Д ок аз а т е ль с т в о п ре д ло же н и я 1. Предположим, что для любого $\varepsilon>0$ существует $q \in(0,1)$ такое, что неравенство (5) выполнено при $t=\left[(1+\varepsilon) a_{T}\right]$ для всех $T \geqslant T_{0}$.

Так как $m_{T}=\min _{0 \leqslant k \leqslant\left[(1+\varepsilon) a_{T_{0}}\right]} \mathbb{P}\left\{\xi(k) \geqslant-\varepsilon b_{T}\right\} \longrightarrow 1$, то $m_{T} \geqslant q$ для всех $T \geqslant T_{1} \geqslant T_{0}$.

Пусть $T \geqslant T_{1}$. Если $\left[(1+\varepsilon) a_{T_{0}}\right] \leqslant k \leqslant\left[(1+\varepsilon) a_{T}\right]$, то $k=\left[(1+\varepsilon) a_{s}\right]$ для некоторого $s \in\left[T_{1}, T\right]$. Так как $b_{T}$ не убывает, то $\mathbb{P}\left\{\xi(k) \geqslant-\varepsilon b_{T}\right\} \geqslant \mathbb{P}\left\{\xi(k) \geqslant-\varepsilon b_{s}\right\} \geqslant q$.

Таким образом, (5) выполнено для $t=k=1,2, \ldots,\left[(1+\varepsilon) a_{T}\right]$. Пусть теперь $k \leqslant t \leqslant k+1 \leqslant\left[(1+\varepsilon) a_{T}\right]+1$. Используя незавйсимость приращений, однородность процесса и тот факт, что $b_{T} \rightarrow \infty$, получим

$$
\begin{aligned}
\mathbf{P}\left\{\xi(t) \geqslant-2 \varepsilon b_{T}\right\} & \geqslant \mathbf{P}\left\{\xi(k) \geqslant-\varepsilon b_{T}\right\} \mathbf{P}\left\{\xi(t)-\xi(k) \geqslant-\varepsilon b_{T}\right\} \\
& \geqslant q \mathbf{P}\left\{\xi(t-k) \geqslant-\varepsilon b_{T}\right\} \geqslant q \mathbb{P}\left\{\inf _{0 \leqslant s \leqslant 1} \xi(s) \geqslant-\varepsilon b_{T}\right\} \geqslant 0.5 q
\end{aligned}
$$

при всех $T \geqslant T_{2} \geqslant T_{1}$. Первое утверждение доказано.

Докажем теперь 2). Используя независимость приращений, однородность процесса и $b_{T} \rightarrow \infty$, получим

$$
\begin{aligned}
& \mathbf{P}\left\{\xi\left(a_{T}\right) \geqslant(1-2 \varepsilon) b_{T}\right\} \geqslant \mathbf{P}\left\{\xi\left(\left[a_{T}\right]\right) \geqslant(1-\varepsilon) b_{T}\right\} \mathbf{P}\left\{\xi\left(a_{T}\right)-\xi\left(\left[a_{T}\right]\right) \geqslant-\varepsilon b_{T}\right\} \\
& \geqslant \mathbf{P}\left\{\xi\left(\left[a_{T}\right]\right) \geqslant(1-\varepsilon) b_{T}\right\} \mathbf{P}\left\{\inf _{0 \leqslant s \leqslant 1} \xi(s) \geqslant-\varepsilon b_{T}\right\} \geqslant 0.5 \mathbb{P}\left\{\xi\left(\left[a_{T}\right]\right) \geqslant(1-\varepsilon) b_{T}\right\}
\end{aligned}
$$

для всех достаточно больших $T$.

Доказ а тельс тв о те о ре мы 3 . Пусть $j \leqslant T<j+1$, где $j \geqslant 2$ и $j / a_{j}>1$. Положим $n_{j}=\left[j / a_{j}\right]-1, I_{k}=\inf _{a_{j} \leqslant s \leqslant a_{j+1}}\left(\xi\left(k a_{j}+s\right)-\xi\left(k a_{j}\right)\right), k \in K$, где $K=\left\{0,2, \ldots, 2\left[n_{j} / 2\right]\right\}$. Ясно, что

$$
U_{T} \geqslant \max _{k \in K} I_{k}=M_{j} .
$$

Так как $a_{j} / j \geqslant a_{j+1} /(j+1)$, то $0 \leqslant a_{j+1}-a_{j} \leqslant a_{j} / j \leqslant 1$ и $k a_{j}+a_{j+1} \leqslant(k+1+$ $1 / j) a_{j}<(k+2) a_{j}$. Поэтому

$$
\begin{aligned}
p_{j} & =\mathbf{P}\left\{M_{j} \leqslant(1-2 \varepsilon) b_{j}\right\}=\left(\mathbf{P}\left\{I_{0} \leqslant(1-2 \varepsilon) b_{j}\right\}\right)^{\left[n_{j} / 2\right]+1} \\
& \leqslant \exp \left\{-\left(\left[\frac{n_{j}}{2}\right]+1\right) \mathbf{P}\left\{I_{0} \geqslant(1-2 \varepsilon) b_{j}\right\}\right\} .
\end{aligned}
$$

Здесь мы использовали независимость приращений и однородность $\xi(t)$. Снова используя эти свойства, имеем

$$
\begin{aligned}
& \mathbf{P}\left\{I_{0} \geqslant(1-2 \varepsilon) b_{j}\right\} \geqslant \mathbf{P}\left\{\xi\left(a_{j}\right) \geqslant(1-\varepsilon) b_{j}\right\} \mathbf{P}\left\{\inf _{a_{j} \leqslant s \leqslant a_{j+1}}\left(\xi(s)-\xi\left(a_{j}\right)\right) \geqslant-\varepsilon b_{j}\right\} \\
& =\mathbf{P}\left\{\xi\left(a_{j}\right) \geqslant(1-\varepsilon) b_{j}\right\} \mathbf{P}\left\{\inf _{0 \leqslant s \leqslant a_{j+1}-a_{j}} \xi(s) \geqslant-\varepsilon b_{j}\right\} \\
& \quad \geqslant \mathbf{P}\left\{\xi\left(a_{j}\right) \geqslant(1-\varepsilon) b_{j}\right\} \mathbf{P}\left\{\inf _{0 \leqslant s \leqslant 1} \xi(s) \geqslant-\varepsilon b_{j}\right\} \geqslant 0.5 \mathbf{P}\left\{\xi\left(a_{j}\right) \geqslant(1-\varepsilon) b_{j}\right\}
\end{aligned}
$$

для всех достаточно больших $j$. Принимая во внимание (7), получим

$$
p_{j} \leqslant \exp \left\{-0.5 H_{3}\left(\left[\frac{n_{j}}{2}\right]+1\right) \exp \left\{-(1-\tau) \beta_{j}\right\}\right\}
$$

для всех достаточно больших $j$. Так как $\ln \ln T=o\left(\ln \left(T / a_{T}\right)\right)$, то ряд $\sum_{j} p_{j}$ сходится. По лемме Бореля-Кантелли с вероятностью 1 неравенство $M_{j}>(1-2 \varepsilon) b_{j}$ выполнено для всех достаточно больших $j$. Учитывая, что $b_{j+1} \sim b_{j}$ при $j \rightarrow \infty$, заключаем, что с вероятностью 1 неравенство $U_{T}>(1-2 \varepsilon) b_{T}$ выполнено для всех достаточно больших $T$. Теорема 3 доказана.

Теоремы 5 и 6 являются следствиями теоремы 4 и формулы для $b_{T}$. Условие (3) легко проверяется. Условие (5) для устойчивых законов, очевидно, выполнено. В теореме 6 условие (5) вытекает из предложения 1 и слабого закона больших чисел для н.о.р. случайных величин. Неравенство (7) проверяется с помошью замечания 2 й леммы 6 из [9]. 
Теорема 7 следует из теоремы 4 , формулы для $b_{T}$ и выпуклости вверх функции $\gamma(x)$.

Доказательство теорем 8-11 сводится к выбору усечения $y_{T}\left(\right.$ если $h_{0}=0$ ), вычислению $b_{T}$ и проверке соответствующих условий для найденной нормировки. Вычисления в этом случае ничем не отличаются от вычислений для н.о.р. случайных величин, подробно проделанных в работе автора [9]. Здесь мы опускаем детали.

Д ок азат ель с т в о те о р е мы 12 . В условиях теоремы (см. [9, раздел IV, с. 345]) $b_{n} \sim \mu a_{n}$ при $n \rightarrow \infty$. Если $n \leqslant T<n+1$, то

$$
b_{n} \leqslant b_{T} \leqslant(1+\varepsilon) \mu a_{n+1} \leqslant(1+\varepsilon) \mu \frac{a_{T}}{T}(n+1)
$$

для любого $\varepsilon>0$ при всех достаточно больших $n$. Отсюда с помощью теоремы 4 получаем все, кроме УЗБЧ, соответствуюшего случаю $a_{T}=T$ для всех $T$.

Пусть теперь $a_{T}=T$. Тогда (см. [9, раздел IV, с. 345]) $\xi(n) / n \rightarrow \mu$ п.н. Применяя далее те же аргументы, что и в книге И. И. Гихмана и А. В. Скорохода [15, с. 493-494], получим УЗБЧ для $\xi(t)$.

При доказательстве последней теоремы можно, конечно, сослаться и на УЗБЧ Колмогорова для н.о.р. случайных величин. Мы упомянули работу [9], чтобы подчеркнуть, что и в случае н.о.р. случайных величин УЗБЧ является следствием универсальных теорем. Это означает, что при доказательстве теоремы 12 не были использованы результаты, не являющиеся следствиями универсальных теорем.

\section{СПИСОК ЛИТЕРАТУРЫ}

1. Shepp L.A. A limit law concerning moving averages. - Ann. Math. Statist., 1964, v. 35 , p. $424-428$.

2. Erdös P., Rényi A. On a new law of large numbers. - J. Anal. Math., 1970, v. 23, p. $103-111$.

3. Csörgö S. Erdős-Rényi laws. - Ann. Statist., 1979, v. 7, № 4, p. 772-787.

4. Deheuvels P., Devroye L. Limit laws of Erdős-Rényi-Shepp type. - Ann. Probab., 1987 , v. 15 , № 4, p. 1363-1386.

5. Mason D. M. An extended version of the Erdős-Rényi strong law of large numbers. Ann. Probab., 1989, v. 17, № 1, p. 257-265.

6. Csörgő M., Révész P. Strong Approximations in Probability and Statistics. New YorkLondon: Academic Press, 1981, 284 p.

7. Frolov A. N. On one-sided strong laws for large increments of sums. - Statist. Probab. Lett., 1998, v. 37, № 2, p. 155-165.

8. Фролов А.Н. Об асимптотическом поведении приращений сумм независимых случайных величин. - Докл. РАН, 2000, т. 372, № 5, с. 596-599.

9. Frolov A. N. One-sided strong laws for increments of sums of i.i.d. random variables. Studia Sci. Math. Hungar., 2002, v. 39, № 3-4, p. 333-359.

10. Deheuvels P., Steinebach J. Sharp rates for increments of renewal processes. - Ann. Probab., 1989, v. 17, № 2, p. 700-722.

11. Steinebach J. Strong laws for small increments of renewal processes. - Ann. Probab., 1991, v. 19, № 4, p. 1768-1776.

12. Зинченко Н. М. Асимптотика приращений устойчивых случайных процессов со скачками одного знака. - Теория вероятн. и ее примен., 1987, т. 32, в. 4, с. 793796.

13. Боровков А. А. Теория вероятностей. М.: Наука, 1986, 431 с.

14. Ибрагимов И. А., Линник Ю. В. Независимые и стационарно связанные величины. М.: Наука, 1965, 524 с.

15. Гихман И. И., Скороход А. В. Теория случайных процессов. Т. 2. М.: Наука, 1973, $640 \mathrm{c}$.

16. Петров В. В. Предельные теоремы для сумм независимых случайных величин. М.: Наука, 1987, 317 с. 